\title{
Factores asociados a la intención de consumo de alimentos nativos en estudiantes de una universidad peruana
}

\author{
Factors associated with the intention of consumption of native \\ food in students of a peruvian university
}

\begin{abstract}
RESUMEN
El objetivo del presente estudio fue identificar los factores asociados a la intención de consumo de alimentos nativos en estudiantes de la Universidad Nacional Agraria La Molina, durante el semestre 2019-II, en la ciudad de Lima. Se utilizó la técnica de la encuesta y como instrumento de recolección se utilizó el cuestionario. Se recolectaron 177 encuestas válidas a través de un muestreo probabilístico por conglomerados. Para el contraste de hipótesis se aplicaron dos pruebas estadísticas: la Prueba Chi Cuadrado de Pearson y la Prueba de correlación de Spearman. En ambas pruebas se halló una asociación estadísticamente significativa entre la variable intención de consumo de alimentos nativos y las variables: actitud, norma subjetiva y control percibido sobre el consumo de los mencionados alimentos. Se concluye que la intención de consumir alimentos nativos en la Universidad Nacional Agraria La Molina está relacionada con la actitud, la norma subjetiva y el control percibido sobre realizar dicho comportamiento.
\end{abstract}

Palabras claves: Consumo; alimentos; nativos; ultraprocesados; monocultivos.

\begin{abstract}
The objective of this study was to identify the factors associated with the intention to consume native foods in students of the Universidad Nacional Agraria La Molina, during the 2019-II semester, in the city of Lima. The survey technique was used and the questionnaire was used as a collection instrument. 177 valid surveys were collected through a probability cluster sampling. Two statistical tests were applied: Pearson's Chi Square Test and Spearman's Correlation Test. In both tests, a statistically significant association was found between the variable intention to consume native foods and the variables: attitude, subjective norm and perceived control over the consumption of the aforementioned foods. It is concluded that the intention to consume native foods at the La Molina National Agrarian University is related to the attitude, the subjective norm and the perceived control over performing said behavior.
\end{abstract}

Keywords: Consumption; food; native; ultra-processed; monoculture.

\section{Carmen Oly Elisa Soria} Mendo $^{1}$

carmenoly.soria@unmsm.edu.pe

Edgar Vicente Armas ${ }^{1}$ evicentea@unmsm.edu.pe

Luis Enrique Espinoza
Villanueva $^{2}$

lespinoza@lamolina.edu.pe

${ }^{1}$ Universidad Nacional Mayor de San Marcos. Lima, Perú

${ }^{2}$ Universidad Nacional Agraria La Molina. Lima, Perú

Presentado: 30/12/2020 - Aceptado: 18/03/2021 - Publicado: 17/06/2021

(C) Los autores. Este artículo es publicado por la revista Gestión en el Tercer Milenio de la Facultad de Ciencias Administrativas Universidad Nacional Mayor de San Marcos. Este es un artículo de acceso abierto, distribuido bajo los términos de la licencia Creative Commons Atribución 4.0 Internacional (CC BY 4.0) [https://creativecommons.org/licenses/by/4.0/deed.es] que permite el uso, distribución y reproducción en cualquier medio, siempre que la obra original sea debidamente citada de su fuente original. 


\section{INTRODUCCIÓN}

Para el 2015, la Organización Panamericana de la Salud (OPS) advirtió un sorprendente cambio de patrones de alimentación basados en comidas y platos preparados a partir de alimentos sin procesar o mínimamente procesados por otros que se basan cada vez más en productos ultraprocesados. Esta situación está generando toda una problemática que se traduce en graves consecuencias en la vida y la salud de la población y del planeta.

En ese sentido, Popkin (2020) indica la existencia de una relación estrecha entre el consumo de alimentos ultraprocesados y las tasas de sobrepeso y obesidad. Asimismo, señala la asociación entre un consumo elevado de estos productos y mayores índices de cáncer y mortalidad cardiovascular, mortalidad total, diabetes y un sinfín de enfermedades no transmisibles.

Por su parte, Monteiro et al. (2017) sostiene que con el incremento del consumo de ultraprocesados, se van estableciendo hábitos de consumo uniformes, desplazando las culturas alimentarias auténticas. Asimismo, señala que la producción y consumo de estos productos implica por un lado una excesiva cantidad de basura, tales como botellas, cajas, envolturas y otros envases; y por otro, extensas rutas de transporte lo que causa un empleo excesivo de energía no renovable.

Esta problemática se complica, pues el consumo y producción de ultraprocesados conduce a la expansión de monocultivos industriales ${ }^{1}$ lo que resulta en el acaparamiento de enormes superficies de tierra, deforestación, sobreexplotación del agua y el uso intensivo de plaguicidas.

De acuerdo con Dammert (s.f.) el reemplazo de bosques tropicales con sembríos de palma aceitera tiene graves impactos ambientales en términos de: emisión de gases de efecto invernadero; impactos en los micro climas locales; pérdida de biodiversidad, cuerpos de agua, las dinámicas del fuego, erosión y pérdida de fertilidad de suelos. Por su parte, Villadiego y Castro (2020) aseveran que la deforestación implica además de una difícilmente cuantificable alteración de los ecosistemas, el aumento de

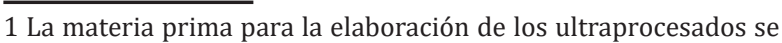
obtiene principalmente de los monocultivos industriales de soja, caña de azúcar y palma de aceite (Villadiego y Castro, 2020). aparición y rápida expansión de enfermedades zoonóticas como la COVID-19. Por otro lado, la apropiación de amplias áreas de tierras, antes en posesión de poblaciones indígenas o campesinas hace que estas deban trasladarse a las ciudades (Carrere, 2001; CONDEG, 2014), otras se han visto obligadas a buscar otras tierras para producir alimentos, lo que supone la deforestación de nuevas tierras (Villadiego y Castro, 2020). Asimismo, "se estima que la explotación agrícola-industrial, cada vez más sustentada en el monocultivo, utiliza hasta diez veces más agua que la agricultura biodiversa" (García, 2009 , p. 2). Esta enorme demanda de agua tiene un profundo impacto "sobre los ecosistemas y sobre las pequeñas comunidades agrícolas" (García, 2009, p.2). Por otro lado, "el uso intensivo de plaguicidas químicos forma parte de los paquetes tecnológicos ligados a los monocultivos" (Bejarano, 2009, p. 1). Castillo et al., (2020) señala que el empleo de plaguicidas en los campos agrícolas repercute en los ecosistemas, contaminando el medio ambiente, suelo, agua y aire. Respecto al uso de glifosato, Rossi (2020) sostiene su vinculación con el cáncer, malformaciones, encefalopatía, autismo, Parkinson y una serie de enfermedades y trastornos en el organismo humano.

En este contexto el consumo de alimentos nativos se constituye como una alternativa, dado que representa un consumo sostenible de acuerdo con la definición propuesta por las $\mathrm{Na}-$ ciones Unidas. Esto es, por un lado, los alimentos nativos pueden cubrir las necesidades básicas de la población y mejorar su calidad de vida, al brindar una alimentación saludable. En ese sentido, el Perú cuenta con una alta variedad de alimentos nativos, de distintos grupos de alimentos, provenientes de su flora megadiversa (Sánchez, 2004 y Cuba et al., 2006); y presentan un alto valor nutritivo (Morón, 1999 y Ayala, G, 2004). Por otro lado, el cultivo de alimentos nativos minimiza el uso de recursos naturales. Siempre que los alimentos nativos provengan de la agricultura campesina, agroecológica o de pequeña escala; pues estas prácticas utilizan los mínimos recursos y son altamente eficientes. En ese sentido, la red campesina usa aproximadamente $10 \%$ de la energía fósil y no más del 20\% del agua que demanda la totalidad de la producción agrícola, con prácticamente cero 
devastación de suelos y bosques (ETC, 2017). Finalmente, el cultivo de alimentos nativos minimiza las emisiones de desechos y contaminantes. Los alimentos nativos son alimentos frescos, sus desechos son orgánicos, los cuales pueden ser aprovechados por otros organismos o transformarse en nutrientes para el medio ambiente.

El presente estudio tiene por finalidad promover el consumo de los alimentos nativos para contribuir a alcanzar el Objetivo 12 de los Objetivos de Desarrollo Sostenible (ODS) que consiste en garantizar modalidades de consumo y producción sostenibles (Hoballah, y Averous, s.f.). Para promover el consumo de alimentos nativos se contempla la formulación de estrategias comerciales basadas en el marketing estratégico, lo que implica un conocimiento profundo de la población. En ese sentido, el estudio tuvo como objetivo conocer los factores que influyen en la intención de consumo de alimentos nativos en una población de estudiantes. La identificación y conocimiento de un mercado potencial permitirá formular estrategias de comercialización adaptadas a sus características y necesidades que posibiliten una aceptación o incremento del consumo del producto.

La presente investigación acerca del consumo de alimentos nativos, resulta ser pionera en el ámbito del estudio del comportamiento de consumo de alimentos. En ese sentido se ha podido encontrar estudios referidos a un tipo de alimento nativo en las que no se hace una distinción como tal. Asimismo, se han hallado investigaciones que abordan el estudio del consumidor en el ámbito de alimentos que cumplen con algunos elementos de la definición de un alimento nativo, entre ellos los alimentos locales y los alimentos tradicionales típicos. Factores psicológicos, socioculturales y de conveniencia estarían asociados al consumo o intención de consumo de alimentos locales, tradicionales o nativos de acuerdo con las investigaciones halladas. En el caso de México, Espejel, Camarena y Sandoval (2014) encontraron que los consumidores prefieren consumir alimentos típicos que le generen una identidad gastronómica, esto es, confianza, costumbre y tradición, pero que a la vez sean productos de alimentación fáciles de encontrar, cocinar y consumir. En el caso de Europa, en Italia,
Visintin, Crovato, Canova, Ravarotto y Capozza (2012) encontraron que las intenciones de consumir alimentos locales por estudiantes de secundaria fueron predichas por aspectos psicológicos y sociales tales como: la actitud, las normas subjetivas y el comportamiento pasado. El consumo de dichos alimentos, se predijo por las intenciones, el control percibido y el comportamiento pasado. No se encontró que el control percibido afecte la intención de consumir alimentos locales. En otro estudio, a nivel de seis países de Europa, Bélgica, Francia, Italia, Noruega, Polonia y España, Pieniak et al. (2009), hallaron que la actitud positiva hacia los alimentos tradicionales, la familiaridad y la importancia de la naturalidad de los alimentos fueron impulsores del consumo de alimentos tradicionales en residentes adultos. Asimismo, la importancia asociada a la conveniencia y la salud actuaron como barreras directas para el consumo de alimentos tradicionales, mientras que la importancia del control de peso surgió como una barrera indirecta al disminuir la actitud positiva hacia los alimentos tradicionales. La importancia del precio al comprar alimentos no se relacionó significativamente con la actitud general y con el consumo de alimentos tradicionales tanto para la muestra agrupada como dentro de cada país, excepto en España. En el Perú, Smith y Trivelli (2001) hallaron que el consumo urbano de alimentos tradicionales en distintas ciudades, estaría relacionado con el estado migratorio de la población. Por su parte, Quispe et al. (2019) hallaron en Arequipa que la comprensión del idioma quechua sería el factor más influyente en el consumo de alimentos andinos en estudiantes de nivel secundario. En Junín, Hinostroza (2018) halló que el consumo de quinua va a depender de factores actitudinales (patrón cultural, gustos y preferencias) tanto en consumidores urbanos como rurales. En cuanto al tarwi, en Puno, Holguín (2018) encontró que su consumo en los pobladores aymaras, estaría relacionado a factores culturales (significados del uso del tarwi) y factores personales (preferencias de consumo). En la población de Ancash, Olórtegui et al. (2010) hallaron que el consumo de tarwi estaría relacionado a los significados sociales, económicos y nutricionales que la población le atribuye al grano. En lo social significaría patrón cultural; en lo económico representaría un generador de producción 
agraria y ganancia; en lo nutricional significa riqueza gastronómica.

En el aspecto teórico, el presente estudio, buscó evaluar la confiabilidad del instrumento formulado y conocer el poder de predicción de la teoría respecto a la intención de consumo de alimentos en el ámbito estudiantil en una universidad de Lima. Respecto al aspecto práctico, la investigación busca promover un mayor consumo de alimentos nativos y la producción campesina o la agroecología- como consumo y producción sostenibles -para contribuir a resolver una amplia gama de problemas, relacionados a la economía de pequeños productores, la nutrición, salud y cultura de la población, y el cuidado y conservación del planeta. Y más aún para hacer frente a las repercusiones de la pandemia de la COVID-19. En ese sentido, el Comité de Seguridad Alimentaria Mundial de las Naciones Unidas (CSA) sostiene la necesidad de un cambio radical de enfoques en favor de la agricultura y los sistemas alimentarios sostenibles (CSA, 2020).

El objetivo general del presente estudio fue identificar los factores asociados a la intención de consumo de alimentos nativos en estudiantes de la Universidad Nacional Agraria La Molina, durante el semestre 2019-II, en la ciudad de Lima, aplicando la Teoría del Comportamiento Planificado (TPB, por sus siglas en inglés). En esa línea, en primer lugar, se buscó definir la asociación de la actitud hacia el consumo de alimentos nativos con la intención de consumo de dichos alimentos. En segundo lugar, se buscó verificar la asociación de la norma subjetiva acerca del consumo de alimentos nativos con la intención de consumo de dichos alimentos. Por último, se buscó comprobar la asociación del control percibido sobre el consumo de alimentos nativos con la intención de consumo de dichos alimentos.

\section{MÉTODOS}

La presente investigación tuvo un diseño no experimental, transeccional de tipo correlacionalcausal. La población de estudio ascendió a 551 estudiantes matriculados en el curso de cálculo integral en el semestre 2019-II en la Universidad Nacional Agraria; para un tamaño de muestra mínimo de 161 estudiantes. Se recolectaron 177 encuestas válidas. Para la selección de las unidades de análisis se consideró el muestreo probabilístico por conglomerados. En ese sentido, se consideró a las aulas o grupos de estudio como conglomerados (unidades muestrales primarias), y a los estudiantes como las unidades más simples (unidades muestrales secundarias). Se utilizó la técnica de la encuesta personal-cara a cara y como instrumento de recolección, un cuestionario en escala Likert de cinco puntos. La primera versión del cuestionario fue formulada con base a la información obtenida en una fase exploratoria y siguiendo las pautas recomendadas por Ajzen (2019a, 2019b). Las preguntas del instrumento se dirigieron a medir los cuatro constructos principales de la Teoría del Comportamiento Planificado: actitud, norma subjetiva, control percibido e intención de consumo de alimentos nativos. Se incluyeron también preguntas de conocimiento y demográficas. Esta primera versión fue revisada y corregida por dos expertos, para luego ser aplicada a una muestra piloto de 15 estudiantes. Posteriormente luego de recolectar y analizar la información, se formuló la versión final del cuestionario, siguiendo las recomendaciones de Ajzen (2019a, 2019b).

La validación del instrumento se realizó a través del análisis de validez de constructo por el método del Análisis Factorial. Dado que se buscó confirmar el modelo de estructura factorial, especificado previamente y basado en un fundamento teórico, se aplicó el Análisis Factorial Confirmatorio (AFC) (Morales, 2011). Dada la naturaleza ordinal de las variables, el AFC se realizó sobre una matriz de correlaciones policóricas entre los ítems (Lloret, Ferreres, Hernández y Tomás, 2014; Miranda, Riquelme, Cifuentes, y Riquelme, 2014) con el Software Estadístico R. Los resultados del AFC confirmaron la estructura factorial propuesta. Respecto los indicadores descriptivos se halló que el modelo teórico se ajustaba adecuadamente a los datos de la muestra. Ver Tabla 1.

Respecto a la Prueba de Hipótesis y la pertenencia de las variables propuestas con su respectiva dimensión, se halló que los niveles de significación calculados fueron en todos los casos menores a 0.05 . Por lo tanto, se confirmó que las variables consideradas pertenecen a sus dimensiones respectivas. Respecto al análisis de las correlaciones de cada variable con su dimensión, todas las variables presentaron correlación 
significativa (correlaciones altas, mayores a 0.5 ) con sus respectivas dimensiones.

Para evaluar la confiabilidad del instrumento se realizó el análisis de consistencia interna calculando el coeficiente de Alpha de Cronbach. El análisis arrojó un valor del coeficiente Alpha de Cronbach de: 0.871 para la escala que mide la variable de Actitud, 0.543 para la escala que mide la variable de Norma Subjetiva y 0.757 para la escala que mide la variable de Control de Comportamiento, lo que se interpreta como una confiabilidad aceptable para las tres escalas (Hernández et al., 2014). Ver Tabla 2.

\section{RESULTADOS}

Las hipótesis a contrastar fueron: HE1: La intención de consumo de alimentos nativos está asociada con la actitud hacia el consumo de dichos alimentos. HE2: La intención de consumo de alimentos nativos está asociada con la norma subjetiva acerca del consumo de dichos alimentos. HE3: La intención de consumo de alimentos nativos está asociada con el control percibido sobre el consumo de dichos alimentos. Se aplicaron dos pruebas estadísticas: la Prueba Chi Cuadrado de Pearson y la Prueba de correlación de Spearman.

Para la hipótesis HE1, se consideró Hnula: No existe una asociación entre la intención de consumo de alimentos nativos y la actitud hacia su consumo. Halternativa: Existe una asociación entre la intención de consumo de alimentos nativos y la actitud hacia su consumo. Para un nivel de significación crítico igual a 0.05 ( $\alpha \mathrm{c}=$ 0.05) y un tamaño de muestra de 177 ( $\mathrm{n}=177)$.

En cuanto a la prueba de Chi Cuadrado de Pearson nos arrojó un nivel de significación calculado de 0.000 menor que el nivel de significación crítico 0.05 . Por lo que se encontró que existe una asociación estadísticamente significativa entre la variable actitud hacia el consumo de alimentos nativos y la intención de consumo de dichos alimentos. Los resultados se aprecian en la Tabla 3.

Tabla 1

Indicadores descriptivos del Análisis Factorial Confirmatorio

\begin{tabular}{|c|c|c|}
\hline \\
\hline \multicolumn{3}{|l|}{ CFI (Índice comparativo ajustado) } \\
\hline TLI (Índice Tucker-Lewis) & 0.98 & $>\mathrm{a} 0.95$ \\
\hline RMSEA (Raíz cuadrada media del error) & 0.058 & $<\mathrm{a} 0.06$ \\
\hline SRMR (Residual raíz cuadrada media del error estandarizado) & 0.057 & $<\mathrm{a} 0.08$ \\
\hline
\end{tabular}

Nota. Elaboración propia.

Tabla 2

Análisis de Confiabilidad de las Escalas

\begin{tabular}{lcc}
\hline Variables & Elem. & $\alpha$ Cronbach \\
\hline X1: Actitud hacia el Consumo de Alimentos Nativos & 8 & 0.871 \\
\hline X2: Norma Subjetiva acerca del Consumo de Alimentos Nativos & 3 & 0.543 \\
\hline X3: Control Percibido sobre el Consumo de Alimentos Nativos & 2 & 0,757 \\
\hline
\end{tabular}

Nota. Elaboración propia.

Tabla 3

Pruebas de chi-cuadrado

\begin{tabular}{lccc}
\hline & Valor & Df & Significación asintótica (bilateral) \\
\hline Chi-cuadrado de Pearson & $71,456^{\mathrm{a}}$ & 12 &, 000 \\
\hline Razón de verosimilitud & 36,126 & 12 &, 000 \\
\hline Asociación lineal por lineal & 19,475 & 1 &, 000 \\
\hline N de casos válidos & 177 & & \\
\hline
\end{tabular}

Nota. a. 11 casillas $(55,0 \%)$ han esperado un recuento menor que 5 . El recuento mínimo esperado es ,02. Elaboración propia. 
En cuanto a la prueba de correlación de Spearman nos arrojó un nivel de significación calculado de 0.000 siendo este menor que el nivel de significación crítico 0.05 , por lo que se encontró que existe una asociación estadísticamente significativa entre la variable actitud hacia el consumo de alimentos nativos y la intención de consumo de dichos alimentos. Los resultados se aprecian en la Tabla 4.

Para la hipótesis HE2, se consideró Hnula: No existe una asociación entre la intención de consumo de alimentos nativos y la norma subjetiva acerca de su consumo. Halternativa: Existe una asociación entre la intención de consumo de alimentos nativos y la norma subjetiva acerca de su consumo. Para un nivel de significación crítico igual a $0.05(\alpha c=0.05)$ y un tamaño de muestra de $177(n=177)$.
En cuanto a la prueba de Chi Cuadrado de Pearson esta nos arrojó un nivel de significación calculado de 0.028 , siendo este menor que el nivel de significación crítico 0.05 , por lo que se encontró que existe una asociación estadísticamente significativa entre la variable norma subjetiva acerca del consumo de alimentos nativos y la intención de consumo de dichos alimentos. Ver Tabla 5.

En cuanto a la prueba de correlación de Spearman esta nos arrojó un nivel de significación calculado de 0.003 siendo este menor que el nivel de significación crítico 0.05 , por lo que se encontró que existe una asociación estadísticamente significativa entre la variable norma subjetiva acerca del consumo de alimentos nativos y la intención de consumo de dichos alimentos. Ver la Tabla 6.

Tabla 4

Correlaciones

\begin{tabular}{|c|c|c|c|c|}
\hline & & & Intención de consumo & Actitud \\
\hline \multirow{6}{*}{ Rho de Spearman } & \multirow{3}{*}{$\begin{array}{l}\text { Intención } \\
\text { consumo }\end{array}$} & Coeficiente de correlación & 1,000 &, $333^{* *}$ \\
\hline & & Sig. (bilateral) & . & 000 \\
\hline & & $\mathrm{N}$ & 177 & 177 \\
\hline & \multirow{3}{*}{ Actitud } & Coeficiente de correlación & ,333** & 1,000 \\
\hline & & Sig. (bilateral) &, 000 & . \\
\hline & & $\mathrm{N}$ & 177 & 177 \\
\hline
\end{tabular}

Nota. ${ }^{* *}$. La correlación es significativa en el nivel 0,01 (bilateral). Elaboración propia.

Tabla 5

Pruebas de chi-cuadrado

\begin{tabular}{llll}
\hline & Valor & Df & Significación asintótica (bilateral) \\
\hline Chi-cuadrado de Pearson & $22,997^{\mathrm{a}}$ & 12 &, 028 \\
\hline Razón de verosimilitud & 21,827 & 12 &, 040 \\
\hline Asociación lineal por lineal & 5,773 & 1 &, 016 \\
\hline N de casos válidos & 177 & & \\
\hline
\end{tabular}

Nota. a. 14 casillas $(70,0 \%)$ han esperado un recuento menor que 5. El recuento mínimo esperado es ,16. Elaboración propia.

Tabla 6

Correlaciones

\begin{tabular}{|c|c|c|c|c|}
\hline & & & Intención consumo & Norma subjetiva \\
\hline \multirow{6}{*}{ Rho de Spearman } & \multirow{3}{*}{$\begin{array}{l}\text { Intención } \\
\text { consumo }\end{array}$} & Coeficiente de correlación & 1,000 &, $223^{* *}$ \\
\hline & & Sig. (bilateral) & . & ,003 \\
\hline & & $\mathrm{N}$ & 177 & 177 \\
\hline & \multirow{3}{*}{$\begin{array}{l}\text { Norma subje- } \\
\text { tiva }\end{array}$} & Coeficiente de correlación & $223^{* *}$ & 1,000 \\
\hline & & Sig. (bilateral) & ,003 & . \\
\hline & & $\mathrm{N}$ & 177 & 177 \\
\hline
\end{tabular}

Nota. ${ }^{* *}$. La correlación es significativa en el nivel 0,01 (bilateral). Elaboración propia 
Para la hipótesis HE3, se consideró Hnula: No existe una asociación entre la intención de consumo de alimentos nativos y el control percibido sobre su consumo. Halternativa: Existe una asociación entre la intención de consumo de alimentos nativos y el control percibido sobre su consumo. Para un nivel de significación crítico igual a $0.05(\alpha c=0.05)$ y un tamaño de muestra de $177(\mathrm{n}=177)$.

En cuanto a la prueba de Chi Cuadrado de Pearson nos arrojó un nivel de significación calculado de 0.009 , siendo este menor que el nivel de significación crítico 0.05 , por lo que se encontró que existe una asociación estadísticamente significativa entre la variable control percibido sobre el consumo de alimentos nativos y la intención de consumo de dichos alimentos. Ver Tabla 7.

En cuanto a la prueba de correlación de Spearman nos arrojó un nivel de significación calculado de 0.013 siendo este menor que el nivel de significación crítico 0.05 , por lo que se encontró que existe una asociación estadísticamente significativa entre la variable control percibido sobre el consumo de alimentos nativos y la intención de consumo de dichos alimentos. Ver Tabla 8.

\section{DISCUSIÓN}

Los resultados obtenidos en el presente artículo, mostraron que la intención de consumo de alimentos nativos en la Universidad Nacional Agraria La Molina está relacionada con la actitud, la norma subjetiva y el control percibido sobre dicho comportamiento.

En cuanto al primer factor, la actitud hacia el consumo de alimentos nativos, se halló una asociación significativa entre esta variable y la intención de consumir dichos alimentos. Este hallazgo va de acuerdo a lo encontrado por Visintin et al. (2012) al observar que la intención de consumo de alimentos locales en Trentino Italia fue predicha por la actitud hacia el consumo de estos productos. Los resultados hallados en cuanto a la primera dimensión de la actitud, indican que existe predominantemente una actitud instrumental muy positiva frente a dicho comportamiento, atribuyéndosele principalmente el adjetivo de muy beneficioso. En cuanto a la segunda dimensión de la actitud, los resultados indican que existe predominantemente una actitud experiencial también muy positiva frente al consumo de alimentos nativos, atribuyéndosele principalmente el adjetivo de muy valioso. En ese sentido, estos resultados

Tabla 7

Pruebas de chi-cuadrado

\begin{tabular}{lccc}
\hline & Valor & Df & Significación asintótica (bilateral) \\
\hline Chi-cuadrado de Pearson & $26,709^{\mathrm{a}}$ & 12 &, 009 \\
\hline Razón de verosimilitud & 23,850 & 12 &, 021 \\
\hline Asociación lineal por lineal & 4,862 & 1 &, 027 \\
\hline N de casos válidos & 177 & & \\
\hline
\end{tabular}

Nota. a. 13 casillas $(65,0 \%)$ han esperado un recuento menor que 5. El recuento mínimo esperado es ,11. Elaboración propia.

Tabla 8

Correlaciones

\begin{tabular}{|c|c|c|c|c|}
\hline & & & Intención consumo & Control percibido \\
\hline \multirow{6}{*}{ Rho de Spearman } & \multirow{3}{*}{ Intención consumo } & Coeficiente de correlación & 1,000 &, $187^{*}$ \\
\hline & & Sig. (bilateral) & . & ,013 \\
\hline & & $\mathrm{N}$ & 177 & 177 \\
\hline & \multirow{3}{*}{ Control percibido } & Coeficiente de correlación &, $187^{*}$ & 1,000 \\
\hline & & Sig. (bilateral) & ,013 & . \\
\hline & & $\mathrm{N}$ & 177 & 177 \\
\hline
\end{tabular}

Nota. * La correlación es significativa en el nivel 0,05 (bilateral). Elaboración propia. 
podrían ser explicados por lo hallado en la fase exploratoria del estudio, que tienen que ver con las creencias de los estudiantes. Frases como: "mejor nutrición", "mayor rendimiento" "consumir lo nuestro", "apoya a agricultores", "buen sabor", "son parte de nuestra cultura", entre otras, relacionados al consumo de alimentos nativos, fueron respuestas abiertas de los estudiantes a las preguntas planteadas. Para definir los aspectos a abordar en las estrategias de comunicación en cuanto a las actitudes, se recomienda investigar las creencias de los estudiantes acerca del impacto que tendría el consumo de alimentos nativos sobre el rendimiento académico, la salud, la identidad cultural, el hedonismo, la conservación del ambiente y la situación económica de los agricultores.

En cuanto a la norma subjetiva, los resultados indican una asociación significativa entre este factor y la intención de consumir alimentos nativos. Los resultados coinciden con la investigación realizada por Visintin et al. (2012) al hallar que las normas subjetivas de los consumidores de Trentino fueron factores predictores estadísticamente significativos de las intenciones de consumir alimentos locales. Los resultados hallados en cuanto a la primera dimensión de la norma subjetiva, indican que existe predominantemente una norma subjetiva de mandato aprobatoria acerca del consumo de alimentos nativos. En cuanto a la segunda dimensión, se observa que existe predominantemente una norma subjetiva descriptiva muy aprobatoria acerca del consumo de los alimentos del estudio. Para la formulación de estrategias de comunicación respecto a la norma subjetiva, se recomienda desarrollar investigaciones para identificar los individuos o grupos que pueden influir en los estudiantes tales como la familia, los amigos, los docentes, autoridades y otros posibles referentes.

Respecto a la tercera variable, se halló una asociación significativa entre la intención de consumir alimentos nativos y el control percibido sobre dicho comportamiento. Este resultado difiere de lo observado por Visintin et al. (2012) el cual no encontró un efecto del control percibido sobre la intención de consumir alimentos locales en la ciudad italiana. Estos resultados podrían deberse a que los estudiantes de secundaria tienen menor capacidad de decisión al elegir sus alimentos en comparación con los estudiantes universitarios. En el caso de los primeros, ellos acceden a sus alimentos principales en sus hogares, en el caso de los universitarios, los compran pues cuentan con un ingreso económico que puede provenir de una mesada de sus padres o por algún trabajo. Los resultados hallados en cuanto a la primera dimensión del control percibido de los estudiantes universitarios, indican que existe predominantemente un control percibido de capacidad alto sobre el consumo de alimentos nativos. En cuanto a la segunda dimensión, se observa que existe predominantemente un control percibido de autonomía también alto sobre el consumo de los alimentos abordados en la presente investigación. Para el desarrollo de estrategias de comunicación en cuanto al control percibido, se recomienda investigar acerca de las habilidades y recursos que facilitarían el consumo de alimentos nativos por parte de los estudiantes.

Los resultados confirman la validez de la teoría en la población de estudio. Por tanto, para promover el consumo de alimentos nativos en la universidad se recomienda formular estrategias de comunicación enfocadas en las actitudes, normas subjetivas y control percibido de los estudiantes.

\section{CONCLUSIONES}

- Se concluye que la intención de consumo de alimentos nativos está asociada con la actitud hacia el consumo de dichos alimentos pues se halló una asociación estadísticamente significativa entre dichas variables en la población de estudio.

- Se concluye que la intención de consumo de alimentos nativos está asociada con la norma subjetiva acerca del consumo de dichos alimentos pues se halló una asociación estadísticamente significativa entre dichas variables en la población de estudio.

- Se concluye que la intención de consumo de alimentos nativos está asociada con el control percibido sobre el consumo de dichos alimentos pues se encontró una asociación estadísticamente 
significativa entre dichas variables en la población de estudio.

\section{REFERENCIAS BIBLIOGRÁFICAS}

Ajzen, I. (2019a). TPB Questionnaire Construction Constructing a Theory of Planned Behaviour Questionnaire. 1-7. https://people.umass. edu/ aizen/pdf/tpb.measurement.pdf

Ajzen, I. (2019b). Sample TpB Questionnaire. 1-9. http://www.people.umass.edu/aizen/pdf/tpb. questionnaire.pdf

Asociación Consejo Nacional de Desplazados de Guatemala - CONDEG (2014). Derechos laborales y derechos de paso en plantaciones de palma africana en Guatemala. In Monocultivos de árboles en América Latina: situación laboral y destrucción de economías locales (p. 53). Movimiento Mundial por los Bosques Tropicales (WRM) Red Latinoamericana Contra los Monocultivos de Árboles (RECOMA). https://wrm.org.uy/ wp-content/uploads/2014/08/empleo-plantaciones-latinoamerica.pdf

Ayala, G. (2004). Aporte de los cultivos andinos a la nutrición humana. En J. Seminario (Ed.), Raíces Andinas: Contribuciones al conocimiento y a la capacitación (pp. 101-112). Universidad Nacional de Cajamarca, Centro Internacional de la Papa, Agencia Suiza para el Desarrollo y la Cooperación. https://nkxms1019hx1xmtstxk3k9sko-wpengine.netdna-ssl.com/wp-content/ uploads/2014/09/07_Aporte_cultivos_andinos_nutric_human.pdf

Bejarano, F. (2009). Monocultivos y agrotóxicos en América Latina. En M. Emanuelli, J. Jonsén y S. Monsalve (Eds.), Azúcar roja, desiertos verdes (pp. 81-86). FIAN Internacional, FIAN Suecia, HIC-AL, SAL. https://hic-al.org/wp-content/ uploads/2019/02/AzucarRoja.pdf

Carrere, R. (2001). Visión de conjunto. Palma aceitera: la expansión de otro monocultivo destructivo. En R. Carrere (Ed.), El amargo fruto de la palma aceitera: despojo y deforestación (pp. 8-12). Movimiento Mundial por los Bosques Tropicales (WRM). https://wrm.org.uy/es/ files/2013/04/El_amargo_fruto_de_la_palma_ aceitera.pdf

Castillo, B., Ruiz, J. O., Manrique, M. A. L. y Pozzo, C. (2020). Contaminación por plaguicidas agrícolas en los campos de cultivos en Cañete (Perú). Revistas ESPACIOS, 41(10), 11-23. http://revistaespacios.com/a20v41n10/a20v41n10p11.pdf

Comité de Seguridad Alimentaria Mundial de las Naciones Unidas. (2020). Recomendaciones del CSA sobre políticas relativas a los enfoques agroecológicos y otros enfoques innovadores en favor de la sostenibilidad de la agricultura y los sistemas alimentarios que mejoran la seguridad alimentaria y la nutrición. Primer borrador. http://www.fao.org/fileadmin/templates/cfs/ Docs1920/Agroecology_an_other_innovative/ NC569_Rev1__CFS_ZERO_DRAFT_AGROECOLOGY_REV1_es.pdf

Cuba, A., Cornejo, C. y Ortega, R. (2006). Sistema de Monitoreo de la Conservación In Situ. http:// www.iiap.org.pe/upload/publicacion/sistema_ monitoreo.pdf

Dammert, J. L. (n.d.). Tráfico de tierras: Deforestación, agricultura de gran escala y titulación en la Amazonía peruana (pp. 1-18). https:// dlc.dlib.indiana.edu/dlc/bitstream/handle/10535/10671/11D3.pdf?sequence=1\&isAllowed=y

Espejel, J. E., Camarena, D. M. J. y Sandoval, S. A. (2014). Alimentos tradicionales en sonora, México: factores que influyen en su consumo. Innovar, 24(53), 127-139. https://doi. org/10.15446/innovar.v24n53.43920

García, A. (2009). Agua y monocultivos: de la explotación agrícola intensiva a la soberanía alimentaria y la seguridad hídrica mundial. En M. Emanuelli, J. Jonsén y S. Monsalve (Eds.), Azúcar roja, desiertos verdes (pp. 95-100). FIAN Internacional, FIAN Suecia, HIC-AL, SAL. al Coalition). https://hic-al.org/wp-content/uploads/2019/02/AzucarRoja.pdf

Grupo ETC. (2017). ¿Quién nos alimentará? La red campesina alimentaria o la cadena agroindustrial. Proyecto 11th Hour. http://www. etcgroup.org/sites/www.etcgroup.org/files/ files/etc-quiennosalimentara-2017-es.pdf

Hernández, R., Fernández, C. y Baptista, M. del P. (2014). Metodología de la Investigación. McGRAW-HILL.

Hinostroza, S. (2018). Factores determinantes del consumo de quinua en el valle del Mantaro y su aporte a la seguridad alimentaria. [Tesis de Pregrado, Universidad Nacional Agraria La Molina]. http://repositorio.lamolina.edu.pe/handle/UNALM/3858

Hoballah, A. y Averous, S. (n.d.). Objetivo 12-Garantizar modalidades de consumo y producción sostenibles: un requisito esencial para el desarrollo sostenible. Naciones Unidas. https://www. un.org/es/chronicle/article/objetivo-12-garantizar-modalidades-de-consumo-y-produccion-sostenibles-un-requisito-esencial-para-el 
Holguin, Z. (2018). Factores que influyen en el consumo del tarwi (Lupinus mutabilis), en el poblador aymara de la zona baja de ILAVE - 2018. Universidad Nacional del Altiplano.

Lloret, S., Ferreres, A., Hernández, A. y Tomás, I. (2014). El análisis factorial exploratorio de los ítems: Una guía práctica, revisada y actualizada. Anales de Psicología, 30(3), 1151-1169. https://doi.org/10.6018/analesps.30.3.199361

Miranda, E., Riquelme, E., Cifuentes, H. y Riquelme, P. (2014). Análisis factorial confirmatorio de la Escala de habilidades sociales en universitarios chilenos. Revista Latinoamericana de Psicología, 46(2), 73-82. https://www.redalyc.org/articulo.oa?id=80532608001

Monteiro C. A., Cannon G. y Moubarac J. C. (2017). Consumo de productos ultraprocesados y la salud humana y del planeta (Public Health Nutrition). IntraMed website: https://www. intramed.net/contenidover.asp?contenidoid $=91804$

Morales, P. (2011). El Análisis Factorial en la construcción e interpretación de tests, escalas y cuestionarios. http://www.upcomillas.es/personal/peter/investigacion/AnalisisFactorial.pdf

Morón, C. (1999). Importancia de los cultivos andinos en la seguridad alimentaria y nutrición. En A. Mujica, J. Izquierdo, J. Marathee, C. Morón y S-E. Jacobsen (Eds.), Memorias: Reunión técnica y taller de formulación de proyecto regional sobre producción y nutrición humana en base a cultivos andinos (pp. 31-46). Organización de las Naciones Unidas para la Agricultura y la Alimentación (FAO), Universidad Nacional del Altiplano Puno- Perú, Universidad Nacional San Agustín Arequipa-Perú, Centro Internacional de la Papa Lima-Perú. https://books.google. com.pe/books?id=u_1DjqZbv-gC\&printsec=frontcover $\& \mathrm{hl}=\mathrm{es} \# \mathrm{v}=$ onepage $\& \mathrm{q} \& \mathrm{f}=$ false

Olórtegui M., R., Paredes A, D., Villafán B., M. y De la Cruz M., C. (2010). El Tarwi (lupinus mutabilis) en Huaraz: aspectos socioeconómicos, nutricionales y culturales. Aporte Santiaguino, 3(1), 125-131. https://doi.org/10.32911/as.2010. v3.n1.430

Organización Panamericana de la Salud. (2015). Alimentos y bebidas ultraprocesados en América Latina: tendencias, efecto sobre la obesidad e implicaciones para las políticas públicas. http://iris.paho.org/xmlui/bitstream/handle/123456789/7698/9789275318645_esp. pdf?sequence $=5$
Pieniak, Z., Verbeke, W., Vanhonacker, F., Guerrero, L. y Hersleth, M. (2009). Association between traditional food consumption and motives for food choice in six European countries. Appetite, 53(1), 101-108. https://doi.org/10.1016/j. appet.2009.05.019

Popkin, B. (2020). El impacto de los alimentos ultraprocesados en la salud. Organización de las Naciones Unidas para la Agricultura y la Alimentación (FAO). http://www.fao.org/3/ca7349es/ CA7349ES.pdf

Quispe, F. y Velasquez, M. (2019). Factores socioculturales que influyen en el nivel de consumo de alimentos andinos en los estudiantes del nivel secundario de instituciones estatales y particulares del distrito de Ttiabaya en el año 2018. Universidad Nacional San Agustín de Arequipa.

Rossi, E. M. (2020). Antología toxicológica del glifosato +1000 Evidencias científicas sobre los impactos del glifosato en la salud, el ambiente y biodiversidad. Naturaleza de Derechos. https://www.conacyt.mx/cibiogem/images/ cibiogem/comunicacion/publicaciones/antologia_toxicologica_glifosato.pdf

Sánchez, I. (2004). Biodiversidad vegetal en los Andes. En J. Seminario (Ed.), Raíces Andinas: Contribuciones al conocimiento y a la capacitación (pp. 39-48). Universidad Nacional de Cajamarca, Centro Internacional de la Papa, Agencia Suiza para el Desarrollo y la Cooperación. https:// nkxms1019hx1xmtstxk3k9sko-wpengine.netdna-ssl.com/wp-content/uploads/2014/09/02_ Biodiversidad_vegetal.pdf

Smith, S. M. y Trivelli, C. (2001). El consumo urbano andinos tradicionales (p. 87). IEP/MSP/ PyMAGROS.

Villadiego, L., \& Castro, N. (2020). Soja: ¿la nueva palma de los biocombustibles? Ecologistas en Acción. https://www.ecologistasenaccion.org/146565

Visintin, E. P., Crovato, S., Falvo, R., Canova, L., Ravarotto, L. y Capozza, D. (2012). Consuming traditional food products of one's province: A test of Planned Behavior Theory. TPM - Testing, Psychometrics, Methodology in Applied Psychology, 19(1), 49-64. https://doi.org/10.4473/ TPM19.1.4 